\section{Transradiale PCl: Häufige Nachwirkungen an Arm und Hand}

Als Gefäßzugang bei perkutanen Koronarinterventionen hat die Arteria radials klare Vorteile. Neue Studiendaten deuten aber auch darauf hin, dass nicht wenige Patienten nach transradialen Kathetereingriffen funktionelle Einschränkungen an Unterarm und Hand verspüren.

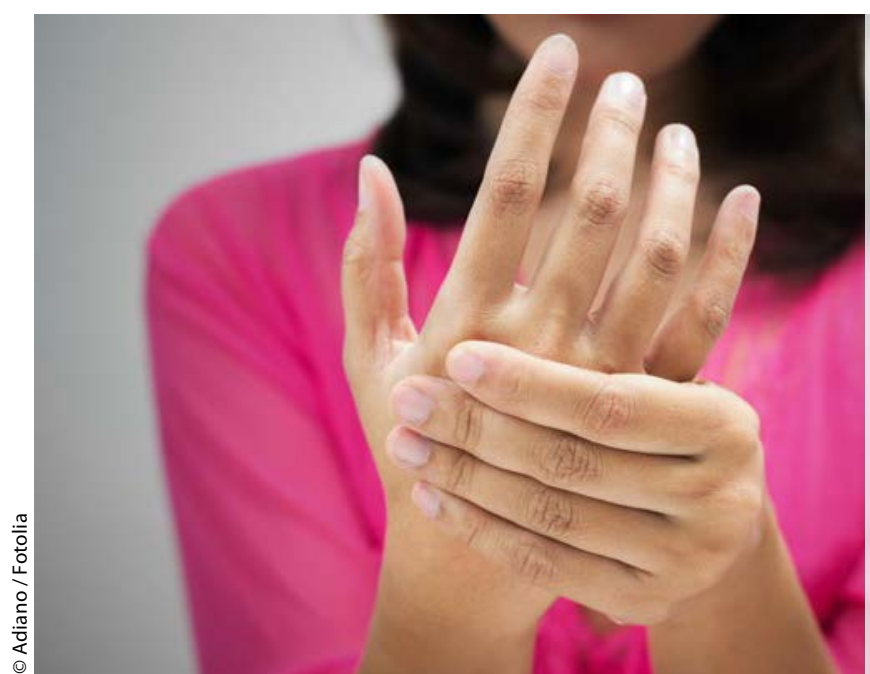

Nach radialer $\mathrm{PCI}$ leiden Patienten des Öfteren an Empfindungsstörungen an den Fingerspitzen oder eingeschränkter Kraft im Handgelenk.
A ls primärer vaskulärer Zugangsweg bei perkutaner Koronarintervention (PCI) wird inzwischen in vielen Ländern die Arteria radialis bevorzugt. Denn große Studien wie MATRIX haben deutliche klinische Vorteile der transradialen gegenüber der transfemoralen PCI offenbart, darunter eine Reduktion von Gesamtmortalität und von schweren Blutungen. Diese Vorteile werden durch eine neue Studie keineswegs infrage gestellt, in der niederländische Untersucher erstmals möglich Folgeprobleme transradialer Koronarinterventionen an der oberen Extremität genauer unter die Lupe genommen haben. Gleichwohl geht aus ersten Zwischenergebnissen dieser Studie hervor, dass knapp $75 \%$ aller $\mathrm{Pa}$ tienten zwei Wochen nach einer PCI via Radialisroute noch immer gewisse funktionelle Beeinträchtigungen an Hand und Unterarm empfanden. Im Vergleich zur nicht punktierten oberen Extremität bestand ein signifikanter Unterschied.

\section{Verminderte Kraft im Handgelenk} Als häufigste Dysfunktionen seien eine eingeschränkte Kraft im Handgelenk,
Paris. Bislang sind 191 Patienten untersucht worden, von denen 143 funktionelle Beschwerden aufwiesen, während 48 beschwerdefrei waren.

Bei 9,8\% aller Patienten mit Dysfunktionen wurden bei der dopplersonografischen Untersuchung Verschlüsse der Arteria radialis festgestellt. Patienten mit funktionellen Einschränkungen waren im Schnitt signifikant jünger als Patienten ohne Problem an Hand und Unter$\operatorname{arm}(63,4$ vs. 67,5 Jahre).

\section{Follow-up über sechs Monate geplant}

Zur Messung von Dysfunktionen an den oberen Extremitäten haben die Studienautoren gemeinsam mit zwei erfahrenen Handchirurgen eigens einen binären Score entwickelt. Damit wurden die per Fragebogen erfassten Symptome und die bei volumetrischen Messungen sowie bei der Prüfung etwa von Griffstärke oder Flexion des Handgelenks erhobenen Befunde quantifiziert.

Die Gruppe um Zwaan plant, insgesamt 500 Patienten in die Studie aufzunehmen. Derzeit ist eine Follow-upDauer von sechs Monaten vorgesehen. Nach Ablauf dieser Zeit wird sich zeigen, ob und wie häufig die in den ersten Wochen nach transradialer PCI verspürten Einschränkungen auch längerfristig bestehen bleiben.

Peter Overbeck
Neue Marke für Pflegekräfte

\section{Zu Springer Medizin kommt jetzt Springer Pflege}

Die Springer-Familie hat Zuwachs bekommen: Seit Februar gibt es neben dem etablierten Bereich Springer Medizin als weitere, eigenständige Sparte Springer Pflege. Unter dem neuen Dach sind die Zeitschrift Heilberufe, das zertifizierte Fortbildungsangebot Pflegekolleg und das Kongressprogramm für Alten- und Krankenpfleger zusammengefasst. Doch damit nicht genug: Weitere Online- und Printangebote sollen folgen, insbesondere wird es einen eigenen Webauftritt www.springerpflege.de geben.

Die Branche steht angesichts des neuen Pflegebedürftigkeitsbegriffs und der Reformen in der Berufsausbildung vor großen Herausforderungen bei der Qualifizierung des Nachwuchses, aber auch bei der Fortbildung erfahrener Pflegekräfte. Hier will das neue Verlagsteam unter Leitung von Falk H. Miekley mit spezifischen e.Learning-Modulen unterstützen. „Die Pflege darf aber nicht losgelöst von anderen Berufsgruppen im Gesundheitswesen betrachtet werden“, lautet Miekleys Einschätzung, „Ärzte und professionell Pflegende müssen eng zusammenarbeiten, wenn sich die Pflegebelange in der Gesellschaft erfolgreich weiterentwickeln sollen." 